\title{
Die gemachten Gäste. Die Bergsteigerdörfer und ihr Bild von den Reisenden
}

\author{
Ruth Weiand \\ DOI: 10.21104/CL.2020.4.04 \\ Constructed Guests. Mountaineering Villages and the Image \\ of a Perfect traveller
}

\begin{abstract}
The Austrian Alpine Association created the brand of Mountaineering Villages to promote a sustainable development of tourism and protect the entire mountain range. The idea of the Mountaineering Villages is the result of the cooperation of alpine associations with local tourist organisations and communities, with hoteliers and committed members of the population. In its policy, the initiative Mountaineering Villages outlines ideas about the ideal guest. It connects the tourists through publications, forms, structures and everyday practices to the villages. The article asks how this relationship develops and what ideas about the guests are created.
\end{abstract}

\section{Keywords}

sustainable tourism, Alpine tourism, sustainable development, guests, vacationscapes, anthropology of policy, tourist relations

\section{Contact}

Ruth Weiand, M. A., Graduate Reseach Group "New Travel - New Media", University of Freiburg, Institute for Cultural Anthropology and European Ethnology, Maximilianstraße 15, 79100 Freiburg, Germany; e-mail: ruth. weiand@neuesreisen.uni-freiburg.de.

\section{Jak citovat / How to cite}

Weiand, Ruth. (2020). Die gemachten Gäste. Die Bergsteigerdörfer und ihr Bild von den Reisenden. Český lid 107, 493-510. doi:http://dx.doi. org/10.21104/CL.2020.4.04 
Der Wunsch nach Veränderung liegt in der Luft. Vorangetrieben von der weltweiten Verbreitung des Corona-Virus wächst die Zahl der Debatten um eine Neuausrichtung der Wirtschaft für die Zukunft. Auch die Urlaubszeit wird von den aktuellen Veränderungen nicht verschont. In einem Interview mit dem Handelsblatt antwortete der Extrembergsteiger und Unternehmer Reinhold Messner auf die Frage, was die Lehre für die Tourismuswirtschaft in den Alpen aus der Corona-Krise sei und wie es weitergehen solle:

„Sowohl die Österreicher als auch wir Südtiroler sollten uns in Europa um die Marktführerschaft mit Blick auf einen sauberen und nachhaltigen Tourismus bemühen. Sie bedeutet auch, auf Menschenmassen in gigantischen Hotels zu verzichten. Ich setze mich dafür ein, dass Südtirol nicht mehr mit alpinen Inszenierungen und Hotspots wirbt, sondern Ruhe und Entschleunigung in den Mittelpunkt stellt." (Siebenhaar 2020)

Gedanken über Nachhaltigkeit und Entschleunigung im Tourismus stehen nicht erst seit dem ersten Auftauchen des Virus zur Diskussion. Die Tourismusbranche beinhaltet eine fragile Balance zwischen Wachstum und Selbstzerstörung: Sie ist einerseits auf das Vorhandensein der sie umgebenden Systeme angewiesen und gleichzeitig tendiert sie dazu, diese zu übernutzen (vgl. Bausinger 1991: 352). Interessant an den Forderungen Messners für einen Tourismus nach der Pandemie ist aber, dass er die gestaltende Rolle der Anbieter*innen und Bereisten innerhalb des Bergtourismus betont. Eine Abkehr von spektakulären baulichen Inszenierungen und Menschenmassen und eine Hinwendung zu Ruhe, Entschleunigung und Nachhaltigkeit wird von Messner auch als Aufgabe der Bereisten gesehen.

Ein Ansatz, um einen solchen zukunftsfähigen Tourismus in den Alpen zu fördern, sind die sogenannten Bergsteigerdörfer. Die länderübergreifende Initiative, gegründet vom Österreichischen Alpenverein, setzt sich nach eigenen Angaben für eine sanfte, naturverträgliche und regionale Entwicklung ihrer Mitgliedsgemeinden ein (vgl. Website der Bergsteigerdörfer: Die Philosophie der Bergsteigerdörfer). Kern des Konzepts der Bergsteigerdörfer ist die Förderung eines nachhaltigen Alpentourismus auf Grundlage der Alpenkonvention, einem völkerrechtlichen Vertrag zwischen den acht Alpenanrainerstaaten und der Europäischen Union zum Schutz und zur Entwicklung des gesamten Gebirgszuges. Auch hier stehen die Anbietenden und Bereisten im Mittelpunkt. In den vom Tourismus abhängigen Bergsteigerdörfern scheint sich meist alles um die Gäste zu drehen, doch 
das Protokoll „Tourismus“ der Alpenkonvention enthält den Grundsatz, auf den sich alle Vertragsparteien geeinigt haben, dass

\begin{abstract}
„die ansässige Bevölkerung in der Lage sein muss [sic], ihre Vorstellungen von der gesellschaftlichen, kulturellen und wirtschaftlichen Entwicklung selbst zu definieren und an deren Umsetzung im Rahmen der geltenden staatlichen Ordnung mitzuwirken" (Protokoll zur Durchführung der Alpenkonvention von 1991 im Bereich Tourismus: 2)
\end{abstract}

Diesem Gedanken folgend, möchte ich hier die Initiative Bergsteigerdörfer ins Zentrum rücken und aus ihrer Perspektive auf die schauen, die sie bereisen.

In der kulturanthropologischen Tourismusforschung überwiegen Studien, die sich mit Tourist*innen und der „Touristenkultur“ (Gyr 1992) beschäftigen. Die Bereisten, vor allem aber die Betrachtung der Verknüpfung von lokalen Strukturen mit größeren Institutionen und Zusammenhängen, fand hier in der Vergangenheit weit weniger Beachtung (vgl. Lauterbach 2006: 55). Der deutsche Kulturwissenschaftler Hermann Bausinger schrieb 1991, dass die Bemühungen um einen sanften Tourismus meist von den Einheimischen ausgingen, sie aber nur erfolgreich sein könnten, wenn auch die touristischen Institutionen und die Tourist*innen involviert wären (vgl. Bausinger 1991: 353). Inwieweit 29 Jahre nach Erscheinen des Aufsatzes Nachhaltigkeit zu einem Trend geworden ist und Bemühungen um sanftes Reisen auch zunehmend von Urlauber*innen ausgehen, sei dahingestellt. Doch es ist dieser von Bausinger angesprochene Dreiklang, der mich interessiert. Es wird im Folgenden also um Institutionen und die durch sie vorgeformten Konzepte, das lokale alltägliche Handeln und Deuten der Bereisten und eine daraus erwachsende Beziehung zu Tourist*innen gehen, wenn ich danach frage, wie die Initiative sich in Beziehung zu Reisenden setzt und welche Vorstellungen über die idealen Gäste hierbei entstehen.

Meine Erkenntnisse stützen sich auf zwei Feldforschungsaufenthalte in Hüttschlag im Salzburger Land und Ramsau bei Berchtesgaden, die ich mit einer Analyse des Informationsmaterials rund um die Bergsteigerdörfer und interner Dokumente zusammendenke. Dem britischen Anthropologen Cris Shore folgend, verstehe ich das Programm, durch das die Bergsteigerdörfer agieren, als deren policy. Als policy sieht Shore all jene sozialen und politischen Blaupausen, mit Hilfe derer ,institutions seek to act upon the world and to manage, regulate or change society" (Shore 2012: 110). In den Bergsteigerdörfern sind die Alpenvereine und ihre Mitarbeiter*innen, die regionalen Tourismusorganisationen und ihre Belegschaft sowie die lokale Bevölkerung die Gestaltenden. Sie alle produzieren kontinuierlich die po- 
licy der Bergsteigerdörfer durch alltägliche Handlungen und Deutungen, durch (verschriftlichte) Konzepte, Werbung und weitere Veröffentlichungen. Ich habe mir für diesen Artikel das Element der Gastgeber*innen Gäste-Beziehung aus diesem Programm herausgegriffen.

Als Peter Haßlacher, damals Mitarbeiter beim Österreichischen Alpenverein und Vertreter der österreichischen Umweltorganisationen im Gremium der Alpenkonvention, das Projekt Bergsteigerdörfer konzipierte, wollte er etwas schaffen, das die Werte der Alpenkonvention verkörpert. So beschrieb es Haßlacher, als ich ihn im Frühling 2019 in seiner Wohnung in Innsbruck besuchte. Die Naturschützer*innen im Alpenverein wären bis dahin immer nur die Verhinderer gewesen. Gegen Wasserspeicher, gegen große Skischaukeln, gegen weitere Seilbahnen, aber ohne eigene Ideen (vgl. Interview vom 13.05.2019). Die Bergsteigerdörfer sehen sich heute als greifbare Umsetzung der textlastigen Alpenkonvention, als kleine und ruhige Orte, eng verbunden mit den Alpenvereinen, die sowohl ein touristisches Angebot bereitstellen wie auch als regionale Entwicklungszentren jenseits des touristischen Treibens wirken (vgl. Österreichischer Alpenverein 2018a: 6; Website der Bergsteigerdörfer: Die Philosophie der Bergsteigerdörfer). Die Initiative setzt sich im Kern einerseits aus den alpinen Vereinen ${ }^{1}$ und ihren für das Projekt zuständigen Mitarbeiter*innen sowie andererseits aus lokalen Tourismusorganisationen, Gemeindevertreter*innen, Betreibenden eines Partnerbetriebes der Initiative sowie engagierten Bürger*innen der Gemeinden zusammen. Es ist das an manchen Punkten auch gegenläufige oder sich widersprechende Zusammenwirken dieser Akteursgruppen, das die Initiative der Bergsteigerdörfer bildet, die ich als „Policy assemblage“ (Ureta 2014), bestehend aus vorgeformten Konzepten, spezifischen Wissensbeständen und alltäglichen Praktiken und Deutungen, verstehe, die stetig zwischen einer organisatorisch institutionellen und einer lokalen Ebene zirkulieren. Mittlerweile ${ }^{2}$ haben sich 29 Dörfer und Gemeinden in vier Ländern und mit teilweise sehr unterschiedlichen Ausgangsbedingungen unter dem Siegel zusammengefunden und gestalten hier jeden Tag das Projekt mit.

Es mag stimmen, dass zu Beginn des Alpinismus Mitte des 19. Jahrhunderts (vgl. hierzu Scharfe 2007) und der Entdeckung der Berge als beliebte Urlaubsdestination der Kontakt mit den Reisenden und ihr Einfluss auf die Lebens- und Wirtschaftsformen der lokalen Bevölkerung eher unwesentlich

1 Die Initiative Bergsteigerdörfer gibt es zum Zeitpunkt der Fertigstellung dieses Artikels in vier Ländern, Österreich, Deutschland, Italien und Slowenien. An ihrer Entwicklung und Umsetzung sind federführend der Österreichische Alpenverein wie auch der Deutsche Alpenverein, der Alpenverein Südtirol, der Club Alpino Italiano und der Planinska zveza Slovenije beteiligt.

2 Stand 05.06.2020. 
waren (vgl. Antonietti 2000: 144). Doch spätestens nach Ende des Zweiten Weltkrieges hängen der Tourismus, der Alpenraum und seine Entwicklungsperspektiven eng miteinander zusammen und in manchen Teilen ist ein gutes Leben für die Einheimischen so fest an die Reisenden gekoppelt, dass diese Verbindung fast schon alternativlos wirkt (vgl. Röthl 2018: 399). Es wäre aber zu kurz gegriffen, in dieser Beziehung zwischen dem Alpenraum und dem Tourismus die dort ansässigen Menschen nur als passive Elemente $\mathrm{zu}$ verstehen, deren Leben zurechtgerückt wird und als Kulisse für die schönste Zeit des Jahres herhalten muss, wie es bei einer oberflächlichen Betrachtung zahlreicher Destinationsmarketingstrategien den Anschein hat (vgl. Stadelmann 1994: 260). In den von mir untersuchten Gemeinden verstehen sich die Bereisten als aktive Gestalter*innen der Urlaubswelt, von der sie unweigerlich ein Teil sind. Gemeinsam mit den alpinen Vereinen kuratieren sie die Bergsteigerdörfer. Während sie dies tun, treten sie in Kontakt mit den dorthin Reisenden, setzen sich unweigerlich zu ihnen in Beziehung und entwerfen ein bestimmtes Bild, das über den Marketingbegriff der „Zielgruppe“ hinausreicht. Die Kulturwissenschaftlerin Adelheid Schrutka-Rechtenstamm versteht die Beziehung zwischen Reisenden und Bereisten als „durch unterschiedliche Erwartungshaltungen hinsichtlich des Kontaktes und vorgeprägte Bilder bestimmt [...]“ (Schrutka-Rechtenstamm 1994: 87), und so hat auch die Initiative der Bergsteigerdörfer Umgangsweisen und Erwartungen, die eine Vorstellung von idealen Gästen entstehen lassen, schon bevor es zu einer tatsächlichen Begegnung kommt. Da es auch Aufgabe der kulturwissenschaftlichen Reiseforschung ist, die vorgefundenen Entwürfe einer Urlaubslandschaft mit all ihren Gestaltungskonzepten und Wertvorstellungen mit Inhalt zu füllen und zu verorten (vgl. Gyr 1992: 24), möchte ich nun schlaglichtartig verschiedene Quellen aus meiner Forschung in den Bergsteigerdörfern herausgreifen und anhand ihrer die Eckpunkte des Verhältnisses zwischen der Initiative und den Gästen der Bergsteigerdörfer skizzieren.

\section{Anbieten: Hüttschlag ${ }^{3}$}

Hüttschlag - „die schönste Sackgasse der Welt" (Feldtagebuch vom 06.03.2020). ${ }^{4}$ Hoch über dem Ort ragt eine große, senkrechte Wand aus verblüffend grünem Gestein empor. Was optisch Eindruck schindet, ist bei

3 Die Beschreibung des Ortes und des touristischen Angebots setzt sich aus meinen Feldnotizen und zahlreichen Gesprächen zusammen, die im Rahmen meiner Feldforschung für mein Dissertationsprojekt im Februar und März 2020 in Hüttschlag entstanden sind.

4 Dieses Zitat fiel in einem Gespräch mit einer Angestellten des Hüttenwirts, einem Partnerbetrieb des Bergsteigerdorfs Hüttschlag. 
den Bewohner*innen Hüttschlags regelmäßig Grund zur Sorge. Der Fels der Hüttschlagerwand besteht aus Grünschiefer, einem bröckligen Gestein, das in Kombination mit der hohen Reliefenergie des Tals immer wieder für Felsstürze, Überschwemmungen und Schlammlawinen sorgt. Im 17. Jahrhundert brachte der Bergbau Wohlstand in die zu dieser Zeit stetig wachsende Gemeinde im Großarltal. Doch der wirtschaftliche Aufschwung hielt nicht an. Ab Mitte des 19. Jahrhunderts wurde es unrentabel, die teilweise über 2000 Meter hoch liegenden Mineralvorkommen in diesem Gebiet abzubauen. Mit dem Niedergang des Bergbaus verarmte das Dorf, das aufgrund seiner Lage, rechts und links von Steilhängen umgeben, kurz vor Talschluss, kaum Möglichkeit für die Ansiedlung größerer Betriebe bietet, die Geld in die Gemeindekassen spülen könnten. Der Beschaffenheit der Umgebung und der finanziellen Situation der Gemeinde geschuldet, konnte in Hüttschlag der Tourismus niemals solche Dimensionen annehmen wie in der Nachbargemeinde Großarl, die Teil des großen Skigebiets Ski amadé ist und über mehrere Liftanlagen und eine Skischaukel nach Dorfgastein verfügt. Hüttschlag setzte anfänglich aus dieser Notlage heraus auf den Wandertourismus und die Sommersaison. 1991 wurde die Gemeinde Teil des Nationalparks Hohe Tauern und seit 1997 teilt sich das ungleiche Paar Großarl-Hüttschlag einen gemeinsamen Tourismusverband, der neben dem Skitourismus verstärkt auf die Bewerbung des gesamten Tals als „Tal der Almen" und somit auf die Sommersaison und das Wandern und Bergsteigen setzt. Hüttschlag war trotz seiner Kooperation mit dem Nachbarn unter den ersten Kandidaten für das Bergsteigerdorfprojekt und bekam 2008 das Siegel überreicht. Mit finanzieller Unterstützung der Initiative wurde ein Jahr später ein Klettersteig in die große grüne Wand gebaut. Etwa zur gleichen Zeit, so berichtet der Alpenvereinsobmann der Sektion Großarl-Hüttschlag, wurde der Wunsch laut, ein dezidiert bergsportlich ausgerichtetes Programm anzubieten, da es bis dato keine Bergführer*innen oder Veranstaltungsunternehmen in Hüttschlag gab und in den Pflichtkriterien der Bergsteigerdörfer aufgeführt ist, dass die Dörfer ein umfangreiches Tourenprogramm anzubieten haben (vgl. Kriterien für Bergsteigerdörfer, Fassung 2014: 3). Der Verein BergGesund wurde geboren. Das Programm von BergGesund passt zur Idee der Bergsteigerdörfer. Die Angebote reichen von einfachen Wanderungen bis zu Eisklettertouren, vom Schneeschuhwandern bis zu kräftezehrenden mehrtägigen Unternehmungen über die Gipfel der Hohen Tauern. Für alle Reisenden scheint hier etwas dabei zu sein. Angeleitet wird von ausgebildeten Bergführer*innen, der Forderung nach Alpinkompetenz (vgl. ebd.) wird so gleich auch nachgekommen. Der Slogan der Bergsteigerdörfer „Bewegung aus eigener Kraft“ (Website der Bergsteigerdörfer) scheint hier passgenau umgesetzt zu werden. 


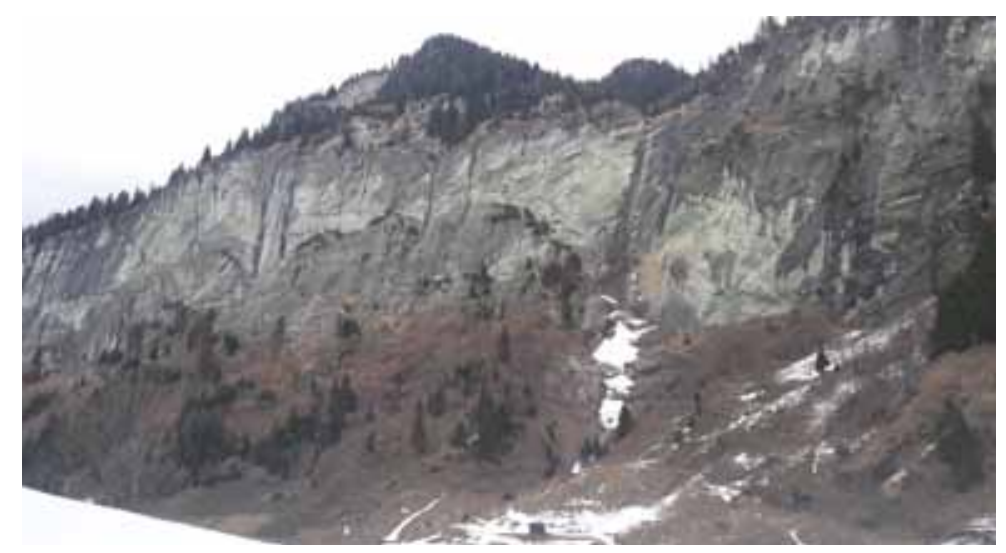

Abb. 1 Hüttschlager Wand (Foto: Weiand)

Die Begegnung im Tourismus zwischen Reisenden und Bereisten ist im Gegensatz zum Großarltal - keine Sackgasse. Die Reisenden lernen im Urlaub Neues kennen und die Bereisten lernen von den Gästen, eine "touristische Perspektive auf ihre Region einzunehmen“, wie die Kulturanthropologin Martina Röthl mit Verweis auf Orvar Löfgren konstatiert (Röthl 2014: 139). In Hüttschlag haben viele Bewohner*innen gelernt, mit den landschaftlichen Voraussetzungen umzugehen und aus den Aktivitäten, denen sie selbst gerne nachgehen, ein umfangreiches touristisches Angebot zu entwickeln. Das Vorhandene - Landschaft, Menschen und Praktiken eingeschlossen - wird dabei nach seiner Konsumierbarkeit bewertet, wird, um den Ausdruck des Soziologen John Urry zu verwenden, unter einen touristischen Blick (tourist gaze) (vgl. Urry 2011: 1-30) genommen und zum Angebot gemacht. Das Angebot in Hüttschlag verrät viel über die Gäste, die in den Ort kommen und kommen sollen. Es richtet sich an Menschen, die körperlich aktiv sein wollen und können. Im Gegensatz zum Nachbarort Großarl gibt es in Hüttschlag kaum andere Möglichkeiten, seine freie Zeit zu gestalten, als an der frischen Luft und in Bewegung.

Das Projekt Bergsteigerdörfer sei etwas für die kleinen Alpengemeinden, die sonst nichts anderes zu bieten hätten, antwortete mir eine Angestellte des Tourismusverbandes Tirol, als ich sie auf einer Messe auf die Initiative ansprach (vgl. Feldtagebuch vom 23.02. 2019). Auch wenn diese Aussage etwas spitz formuliert war, berührt sie doch einen Kerngedanken der Bergsteigerdörfer. Es geht nicht darum, neue touristische Infrastrukturen zu bauen, um die Attraktivität der Dörfer für die Gäste zu steigern, sondern darum, das Vorhandene bewusst und dezent in Szene zu setzen, als Stärke umzudeuten und konsumierbar zu machen. 
Die größte Herausforderung sei, stellt der Gründer von BergGesund fest, „dass [man] das Angebot, das Hüttschlag eigentlich hat, in Bezug auf den Naturliebhaber, den Wanderer, den Bergsteiger, den Skitourengehen und so weiter, dass man das ganz stark in den Vordergrund stellen muss" (Interview vom 03.03.2020). Die Deutung des Vorhandenen und damit zusammenhängend die Ausrichtung des Angebotes in Hüttschlag, aber auch innerhalb der gesamten Initiative, auf den Bergsport geht - so deute ich die Aussage - mit einem bestimmten Bild von den idealen Gästen einher: Es ist der körperlich aktive, ,bergnarrische' Mensch, an den sich die Bergsteigerdörfer wenden, übrigens eine Beschreibung, die auch auf viele Mitglieder der Initiative zutrifft.

\section{Erklären: Ramsau ${ }^{5}$}

Um eine weitere Facette der Beziehung zwischen Initiative und Reisenden zu verstehen, begeben wir uns nach Ramsau bei Berchtesgaden. Nur eineinhalb Stunden von Hüttschlag entfernt, hat das erste deutsche Bergsteigerdorf dennoch ganz andere Voraussetzungen als sein österreichisches Pendant. Es ist das übernachtungsstärkste Dorf innerhalb des Projekts und an schönen Sommertagen stark vom Tagestourismus in Anspruch genommen. Der tiefblaue Königssee, ein Wahrzeichen der bayerischen Alpen, liegt in unmittelbarer Nachbarschaft. Von München aus erreicht man Ramsau in zwei Stunden, vorausgesetzt der Stau auf der Autobahn in Richtung Berge ist an einem Schönwettersonntag nicht allzu lang. Für den Geschäftsführer der Tourismusinformation im Ort waren die Bergsteigerdörfer eine Möglichkeit, in Anbetracht des zunehmenden Andrangs seine eigenen Vorstellungen einer naturnahen Gestaltung des Tourismus ins Blickfeld zu rücken. Nachdem er sich mit den Folgen touristischer Übernutzung auseinandergesetzt hatte, beschloss er, dass er mit „seinem Dorf“ if it's possible: (vgl. Interview vom 07.05.2019) einen anderen Weg gehen möchte, sich auf das konzentrieren möchte, „was unsere wirkliche Stärke" (ebd.) ist. Auch hier steht der Gedanke im Zentrum, mit den lokalen Gegebenheiten zu arbeiten, anstatt mehr touristische Infrastruktur aufzubauen. Seit 2015 ist Ramsau ein Mitglied der Bergsteigerdörfer. Neben einer breiten bergsportlichen Auswahl ist aber noch etwas anderes für den Geschäftsführer wichtig: Bei einem Gespräch zeigt

5 Die Beschreibung des Ortes und des Umgangs mit den Gästen setzt sich aus meinen Feldnotizen und zahlreichen Gesprächen zusammen, die im Rahmen meiner Feldforschung für mein Dissertationsprojekt im Mai 2019 und Oktober 2020 in Ramsau bei Berchtesgaden entstanden sind. 
er mir ein Faltblatt, das die Tourismusinformation in Zusammenarbeit mit einem Ehepaar, das in den Bergen eine Alm betreibt, entworfen hat. Versehen mit spielerisch illustrierten Zeichnungen werden hier zentrale Verhaltensregeln beim Almbesuch aufgeführt. Er erklärt mir:

"[...] das ist ja auch Ansinnen von uns [...], dass man den Leuten einfach ein bisschen hilft, zu entschleunigen und den Wert der Natur schlechthin zu erklären. ,Was ist eine Alm, was geschieht denn da oben?" Auch einem Urlauber, auch einem Einheimischen zu sagen, was soll ich tun, was soll ich vermeiden [...]. Ja, das muss man schon einmal erklären [...], wobei der Tourismus ja suggeriert, eine Alm, wunderschön!' Es gibt Almen, zum Beispiel die Fenneralm, habe ich gestern gehört, die Bergstation der Fennerbahn wird Fenneralm genannt, ein Bauwerk aus Beton, das die Bergstation einer Sesselbahn ist und da ist eine Gastronomie drin. Was hat das mit einer Alm zu tun?" (ebd.)

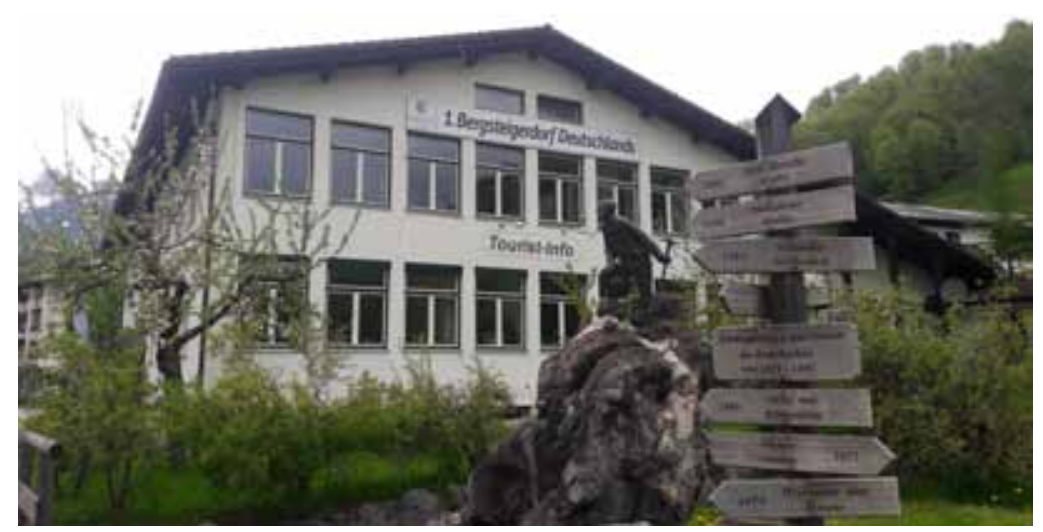

Abb. 2 Rathaus in Ramsau bei Berchtesgaden (Foto: Weiand)

Die Tourismusinformation in Ramsau versucht, mithilfe von Erklärungen, bei deren Zusammenstellung auch andere lokale Akteursgruppen, wie hier die Almbetreibenden, einbezogen werden, die Besucher*innen hin zu einem als richtig erachteten Verhalten zu lenken. Der Kulturanthropologe Orvar Löfgren entwirft den Begriff der vacationscapes, der Urlaubslandschaften, um zu beschreiben, dass die Orte, an die Menschen in ihrer freien Zeit reisen, nicht einfach gegeben sind, sondern durch das $\mathrm{Zu}$ sammenwirken von Erwartungen, Vorannahmen, stabilen Routinen und Gewohnheiten erst entstehen. „It is the interaction between certain landscape characteristics, mindsets, and tourist technologies of movement and 
representation that produces a vacationscape." (Löfgren 1999: 98) Löfgren macht keinen Unterschied zwischen den Perspektiven der Reisenden und der Bereisten, wenn er ausführt, dass eine Urlaubslandschaft aus einer Mischung aus persönlichen Erinnerungen und kollektiven Vorstellungen besteht (vgl. ebd.: 2). In Anlehnung an Löfgren kann der Umgang der Tourismusinformation Ramsau mit ihren Besucher*innen als Versuch gedeutet werden, den von der Tourismusindustrie produzierten und vom Ramsauer Touristiker als falsch gedeuteten Vorstellungen über das Leben und Wirtschaften in den Bergen etwas entgegenzusetzen und über Erklärungen eine andere Sichtweise zu vermitteln. Über die Deutung einer bestimmten Haltung (mindset), die Lenkung der Besucher*innen zu einem „besseren“ Verhalten (tourist technologies of movement) und die Hinterfragung kollektiver, von der Tourismusindustrie geprägter Vorstellungen (representations) versucht die Tourismusinformation in Ramsau, die sie umgebende Urlaubslandschaft zu gestalten. Ihr Geschäftsführer möchte den Besucher*innen eine Hilfestellung anbieten, sich den Sichtweisen der lokalen Bevölkerung, wie beispielsweise der Wertschätzung gegenüber der Natur und den im Urlaubsgebiet wirtschaftenden Menschen, anzunähern. Die Akzeptanz und Unterstellung unter die „Normen und Werteskalen des Gastgebers“, so der Kulturanthropologe Karl Wernhart, würde maßgeblich die Beziehung zwischen Reisenden und Bereisten bestimmen (Wernhart 1997: 29).

Erklärungen statt einfacher Ge- und Verbote erfordern von den Reisenden eine Bereitschaft, sich tiefer mit den Kontexten, die den eigenen Urlaub umgeben, auseinanderzusetzen und auf sie zu reagieren. Es fordert auch dazu auf, ursprüngliche Sichtweisen und vielleicht auch erlernte touristische Praktiken zu hinterfragen. Diese Art der Besucherlenkung setzt voraus, dass die potenziellen Besucher*innen gewillt sind, sich mit der Situiertheit ihres eigenen Urlaubs auseinanderzusetzen und die Auswirkungen ihres eigenen Handelns nicht nur zu verstehen, sondern auch eine gewisse Bereitschaft zur Anpassung mitzubringen. Die Tourismusinformation in Ramsau spricht mit ihrer Vorgehensweise also Reisende an, die lernbereit und einsichtig sind und ihre Rolle in der Gestaltung von Urlaubslandschaften reflektieren und in größere Bezüge einordnen können. Eben solche Besucher*innen wären ideal für das Bergsteigerdorf Ramsau, denn nur dann kann das hier beschriebene Vermittlungskonzept aufgehen.

\section{Annähern: Ein alpenkulinarisches Reisebuch}

Almkassuppe, Spinatknödel, Bachforelle, Buchterln - diese Gerichte sind nur eine kleine Auswahl der Köstlichkeiten, die im alpenkulinari- 
schen Reisebuch der Bergsteigerdörfer (Österreichischer Alpenverein $2018 \mathrm{~b})$ vorgestellt werden. Mit Vor-, Haupt- und Nachspeisen im Gepäck führt es durch das Projekt, stellt die Dörfer und die dort ansässigen Partnerbetriebe vor, und der Verdauung wegen gibt es noch jeweils einen Tourenvorschlag mit an die Hand. Im einleitenden Text, verfasst von Roland Kals, Mitarbeiter des Österreichischen Alpenvereins und seit den Anfangsjahren maßgeblich in das Projekt involviert, heißt es dann weiter:

„Genießen und Verweilen in den Bergsteigerdörfern - da gehören Speis und Trank unbedingt dazu. Wir präsentieren eine bunte Palette von Partnerbetrieben und Schutzhütten, wobei versucht wird, ein wenig hinter die Kulissen zu schauen und auch die Menschen zu porträtieren, die unsere Gastgeber sind. [...] Ein Wort zum Begriff „Bergsteigeressen": Wir verstehen darunter nicht jenes preisgeregelte Gericht, das auf Alpenvereinshütten den Vereinsmitgliedern mit schmalem Geldbeutel vorbehalten ist und früher für eher einfallslose und billige Zutaten bekannt war. Das hat sich in den letzten Fahrzehnten gründlich geändert. Sowohl die Ansprüche wie auch die Kaufkraft der bergsteigenden Menschen sind markant gestiegen [...] Also definieren wir in diesem Büchlein den Begriff Bergsteigeressen einfach anders: Schmack- und nahrhafte Speisen für Menschen, die gerne am Berg unterwegs sind und denen es neben der sorgfältigen Zubereitung auch um Herkunft und Produktionsweise der Grundstoffe geht. Wir leben ja in einer Zeit, in der das Bewusstsein für regional und ethisch einwandfrei produzierte Produkte schon weit verbreitet ist. "(Österreichischer Alpenverein 2018b: 16)

Auf 167 Seiten geht es nun quer durch die Alpen und durch die einzelnen Dörfer. Die Partnerbetriebe werden vorgestellt, die Lesenden erfahren einiges über die Lage der Häuser, ihre Architektur und Geschichte und vor allem über die Gastgeber*innen. Es werden Werdegänge, nicht selten sogar die der letzten Generationen, dargestellt. So werden die Menschen tief in die Geschichte der jeweiligen Ortschaften eingeschrieben. Das Essen scheint hier dann oft gar nicht mehr so zentral zu sein. Die Rezepte werden optisch, sowohl durch Schriftart als auch durch die Fassung in einen extra Kasten, von der Beschreibung der Betriebe getrennt und stehen oft am Ende der Ausführungen. So erfahren die Lesenden, die sich schon zu Beginn als Gäste der Bergsteigerdörfer fühlen können, wenn von „unseren Gastgebern“ gesprochen wird, dass ein Wirt aus Hüttschlag nicht nur ausgebildeter Koch ist, sondern auch gerne Fliegenfi- 
schen geht und „bergnarrisch“ ist (vgl. ebd.: 100-101). Ein Ramsauer Betreiber hat eine Leidenschaft für die alpine Botanik (vgl. ebd.: 43), und seine Kolleg*innen am anderen Ende des Dorfes werden als begeisterte Berggeher*innen beschrieben, die wüssten, „dass der Reiz unserer Bergheimat" (ebd: 96) nur durch eine schonende Nutzung derselben erhalten werden kann. Allgemein wird an mehreren Stellen die Achtung der Wirt*innen vor ihrer Umgebung und ihre Ausrichtung auf regionale und nachhaltig produzierte Erzeugnisse betont.

Das alpenkulinarische Reisebuch bringt den Gästen die verschiedenen Mitgliedsgemeinden und ihre Gastgeber*innen näher. Dabei erzählt es nicht nur über die Wirt*innen, ihre Häuser und ihr kulinarisches Angebot, sondern auch eine Geschichte über deren Beziehung zu ihren Gästen, an die sich wiederum das Buch wendet. Mit dem Blick hinter die Kulissen wird die abstrakte Struktur des Projekts greifbar, es bekommt Gesichter und Geschichten. Doch es sind nicht irgendwelche Geschichten, sondern solche von Gastgeber*innen, die sich um ihre Gäste kümmern wie auch um den Erhalt einer lokalen Wertschöpfungskette. Gastgeber*innen sind hier nicht nur Expert*innen ihrer Umwelt und tief mit ihr verwurzelt, sondern haben, wie auch die Gäste, an die sich dieses Buch richten soll, ein großes „Alpenbewusstsein“ (Leimgruber 2005: 152), das ein Interesse an der Bewahrung der alpinen Natur und Kultur beinhalte (vgl. ebd.). Selbst „bergnarrisch“ und als „begeisterte Berggeher" beschrieben, teilen sie die hohen Ansprüche ihrer Kund*innen. In den Bergsteigerdörfern, so könnte man zugespitzt formulieren, trifft der Gast auf seinesgleichen oder umgekehrt: In den Bergsteigerdörfern suchen die Gastgeber*innen nach Gästen, die die Welt so sehen wie sie.

Essen und Trinken, stellt der Kulturanthropologe Bernhard Tschofen fest, seien nicht nur ein Ausdruck des sozialen Status, sondern zunehmend auch eine symbolische Markierung kultureller Zugehörigkeit (vgl. Tschofen 2010: 27). Und auch das Reisebuch „beweist einmal mehr die kulturelle Dimension des Projekts Bergsteigerdörfer" (Österreichischer Alpenverein 2018b, Vorwort von Ewald Galle: 5). Über Essen und Trinken können nicht nur Regionen konstruiert werden, wie Tschofen in seinem Aufsatz anschaulich zeigt oder auch Timo Heimerdinger konstatiert, wenn er vom Regionalismus in der Küche als Ausdruck einer „regional-räumlichen Orientierung“ (Heimerdinger 2005: 208) spricht, sondern auch Urlaubslandschaften und Beziehungen. Über die Herausstellung gemeinsamer Interessen und geteilter Wertschätzungen durch Gastgeber*innen und Gäste wird im alpenkulinarischen Reisebuch eine gemeinsame Identifikationsebene angeboten, ein Vorschlag über die $\mathrm{Zu}$ gehörigkeit zu einem geteilten kulturellen Wertesystem und einer Vorstel- 
lungswelt gemacht. In den Bergsteigerdörfern steht über der strukturellen Trennung zwischen Gastgeber*innen und Gästen ein angenommenes gemeinsames Bewusstsein. Die Herstellung eines Wir-Gefühls, hinter dem die Annahme eines gemeinsamen Denkens, Fühlen und Wertschätzens steht, lässt beide Seiten zusammenrücken.

\section{Einschreiben: Zu Gast in den Bergsteigerdörfern}

Wie lässt sich nun also die Beziehung, in welche sich die Bergsteigerdörfer zu Tourist*innen setzen, beschreiben? Sie als reines Wechselspiel aus Angebot und Nachfrage auf dem kapitalistischen Tourismusmarkt zu begreifen, wäre aus kulturanthropologischer Sicht zu kurz gegriffen. Vielmehr besteht sie aus der Perspektive der Initiative aus feinen Erzählsträngen, die bei näherer Betrachtung Aussagen über die Vorstellung von idealen Gästen in den Bergsteigerdörfern entstehen lassen. Der Begriff Gäste trägt immer auch etwas Relationales in sich. Wo eine Person $\mathrm{zu}$ Gast ist, setzt sie sich und etwas oder jemand mit ihr in eine Beziehung, die nicht selten eine Tauschbeziehung ist (vgl. Bahr 1997). Über die Angebotsstrukturen und ihre Vermittlung setzen sich die Bergsteigerdörfer in Beziehung zu ihren Gästen. Das touristische Angebot wird aus den lokalen Bedingungen heraus entwickelt und beinhaltet eine Fokussierung auf bergsportliche Aktivitäten. Neben dem körperlich-sportlichen Aspekt spielt aber auch die Bereitschaft der imaginierten Gäste, sich aktiv mit der sie umgebenden Urlaubslandschaft auseinanderzusetzen eine Rolle. Sich informieren, verstehen, sich anpassen, wertschätzen und überdenken scheinen nur einige der Fähigkeiten zu sein, die Gäste der Bergsteigerdörfer mitbringen sollen. Die Bergsteigerdörfer wenden sich an lernbereite, einsichtige Gäste, die komplexe Zusammenhänge begreifen möchten und können. Es wird vermittelt, dass hier Gäste und Gastgeber*innen in Einklang miteinander handeln. Sie können sich mit dem gleichen kulturellen Wertesystem identifizieren, woraus die Annahme eines geteilten (Alpen-) Bewusstseins, einer übereinstimmenden Wertschätzung und gemeinsamer Interessen entsteht. „Spannungsfrei verlaufen die Beziehungen von Besuchern und Besuchten vor allem dann, wenn der soziale und kulturelle Abstand zwischen beiden Gruppen gering ist" schreibt der Soziologe Christoph Hennig in seinem Buch Reiselust (1997: 130). Dieser Befund lässt sich auch auf das Verhältnis der Bergsteigerdörfer zu ihren Gästen übertragen, dem eine positive Bewertung des Tourismus zu Grunde liegt. Immerhin wird er als Entwicklungsmotor der gesamten Initiative und damit auch als Basis einer nachhaltigen zukunftsfähigen Entwicklung im Alpenraum beworben. 


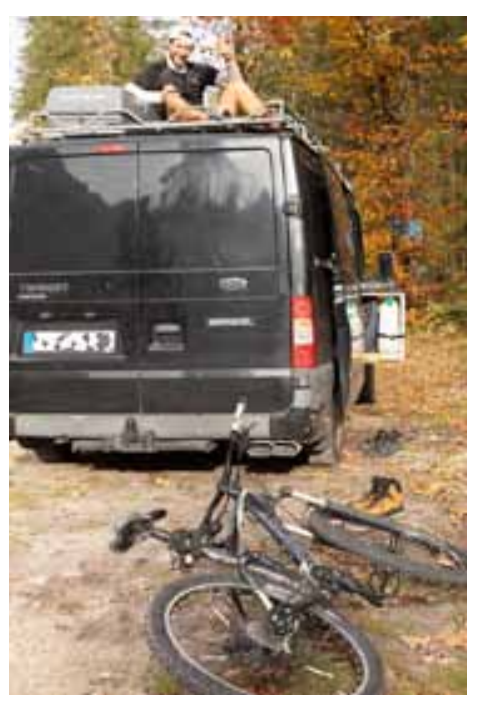

Abb. 3 Zu Gast in den Bergsteigerdörfern (Foto: Weiand)

Das Verhältnis zwischen Gast und Gastgeber*in sei ein „enges integrativ vernetztes, dialektisch ausgelegtes Interaktionsgefüge“" (Wernhart 1997: 29), das im Gegenzug zu Beherbergung und Schutz die Akzeptanz der Gesellschaftsstruktur der Bereisten beinhalte. Die Bergsteigerdörfer stehen in einer gewissen Abhängigkeit von ihren Gästen. Damit das Projekt funktioniert, brauchen sie eben solche, die ihre Werte und Normen anerkennen, die sich für ihr Konzept entscheiden, ohne Zwang und Kontrolle. Hier drückt sich der Wunsch nach dem aus, was der Erziehungswissenschaftler und Tourismusforscher Horst Opaschowski als das Bild des „neuen Touristen“ beschreibt:

\section{„der neue Tourist soll ein einsichtiger, ein konsumkritischer, ein genügsamer und anpassungswilliger, ein rücksichtsvoller, ein innen- geleiteter, ein selbstbeschränkt reisender, ein experimentierfreudiger, kreativer, ein lernbereiter Tourist sein." (Opaschowski 2001: 21)}

Opaschowski sieht dieses gewandelte Bild der Touristin oder des Touristen als Entwicklung, die Hand in Hand mit einem qualitativen Umschwung in der Tourismuskritik ging. Aus einer Kritik der Privilegierten wurde spätestens mit Eingang des Tourismus in die gesellschaftliche Nachhaltigkeitsdebatte der 90er Jahre eine Kritik der Bereisten, wobei die Frage nach dem Verhältnis zwischen Tourismus und Lebensqualität ins Zentrum rückte (vgl. ebd.). Diese Frage beschäftigt auch die Bergsteigerdörfer als regionale Entwicklungsinitiative von Anfang an. Ihre Entstehungsgeschichte, zuerst als Gedanke im Kopf Peter Haßlachers, 
beginnt in den 90er Jahren zu wachsen, und so ist es kaum verwunderlich, dass auch das Bild des "neuen Touristen“ in der Initiative aufgegriffen wird. Zusammengenommen mit der Forderung aus der Alpenkonvention nach einer zwingenden souveränen Handlungsmacht der ansässigen Bevölkerung lässt sich die Beziehung, in welche sich die Bergsteigerdörfer zu ihren Gästen setzten, als eine zwischen zwei mündigen Parteien beschreiben, die sich zwischen Anbieten, Erklären, Annähern und Erwarten bewegt. Das bedeutet auch, dass sie stark von einer beidseitigen Aktivität gekennzeichnet ist. Eine gängige Kritik an Tourist*innen sei, dass sie keinerlei Eigeninitiative zeigen würden und passiv seien, konstatiert Hennig (vgl. Hennig 1997: 17). Genau das Gegenteil imaginieren die Bergsteigerdörfer in Bezug auf Idealvorstellungen von ihren Gästen. Sie grenzen sich damit vielleicht auch wieder ein Stück weit von anderen Destinationen im Alpenraum ab. In den Bergsteigerdörfern sollen die Touristen aktiv sein, nicht nur körperlich, sollen bewusste Entscheidungen treffen und sich Gedanken machen. Das wird erwartet, darüber wird erzählt und dafür wird ein Angebot geschaffen. Die Bereisten und die Alpenvereine treten in den Bergsteigerdörfern als selbstbestimmte Gestalter*innen der Urlaubslandschaft auf. Sie sind es, die die Geschichte der Bergsteigerdörfer schreiben und erzählen, denn so präsent die Reisenden auch sind, im Script der Initiative tauchen die tatsächlichen Urlauber*innen nur als Idealbilder auf. Die Reisenden werden von ihr imaginiert und als Entität mit gewissen Eigenschaften bestimmt und eingeschrieben.

Doch dieses Vorgehen hat auch eine Kehrseite. Der Philosoph HansDieter Bahr schreibt, dass es so scheine, dass ,jeder x-beliebige zum Gast werden kann, der Gast also nur eine Leerstelle bezeichne“ (Bahr 1997: 39). Es könne jede Person, egal wo sie herkomme, wie sie aussehe und was sie mitbringe, zum Gast werden (vgl. ebd.). Was als theoretischer Gedanke und als philosophischer Ansatz sinnvoll erscheint, gilt in seiner Totalität aber nicht in den Bergsteigerdörfern. Hier ist der Platz des Gastes nicht leer, sondern dicht besetzt mit Erwartungen und Vorstellungen.

An den meisten Tagen des Jahres stehen Tourist*innen aus Asien auf der Brücke über die Ramsauer Ache und fotografieren die Pfarrkirche vor dem Bergpanorama. Sie bleiben oft nicht lange, nur für ein Foto, ein Stück Kuchen und eine Brise Bergluft. Die Ramsauer sprechen viel über „die Chinesen", sind stetig in Kontakt mit ihnen, aber im Konzept der Bergsteigerdörfer tauchen sie nicht auf, weder auf Bildern, noch in Textform. Nicht jede* $r$ kann also zum Gast in den Bergsteigerdörfern werden. Die Initiative erwartet von ihren Gästen, sich aus eigener Kraft in den Bergen zu bewegen. Doch dies ist nur denen möglich, die diese Kraft auch haben, die sich so bewegen können, dass sie das bergsportliche Programm wahrnehmen 
können. Mit einem Rollstuhl beispielsweise würden Gäste in den Dörfern nicht weit kommen. Die Idee der Bergsteigerdörfer beinhaltet aber auch die Bestimmung des idealen Gastes als eines lernbereiten, kreativen und konsumkritischen Menschen. Diese Eigenschaften setzen Wissen voraus und die Fähigkeit, es sich selbst anzueignen. Es setzt aber auch voraus, dass große Begrifflichkeiten wie Nachhaltigkeit oder Entschleunigung auf dieselbe Art und Weise aufgeschlüsselt und mit denselben Werten versehen werden, wie es die Initiative tut. Auch Mündigkeit muss erlernt werden, und es wäre eine Illusion zu behaupten, dass alle Menschen die gleichen Startvoraussetzungen mitbringen und Zugriff auf Wissensbestände besitzen, um dies zu tun. Mit ihrer Ausrichtung auf wenig, dafür qualitativ hochwertigen und nachhaltigen Tourismus wirken sie exklusiv.

Natürlich haben die Dörfer keine realen Schranken, „jeder x-beliebige“, um die Worte Bahrs zu verwenden, kann hierhin reisen. Doch nicht zu jedem $x$-Beliebigen setzten sich die Bergsteigerdörfer in Beziehung. Nicht alle können hier zu Gästen werden.

September 2020

\section{Bibliographie}

\section{Quellen}

Feldtagebuch vom 23. 02. 2019.

Feldtagebuch vom 06. 03. 2020.

Kriterien für Bergsteigerdörfer, Fassung 2014. Als pdf abrufbar unter: https://www.bergsteigerdoerfer.org/6-0-Die-Philosphie-der-

Bergsteigerdoerfer.html [Stand 05.06.2020].

Österreichischer Alpenverein (Hg.). 2018a. Kleine und feine Bergsteigerdör-

fer zum Genießen und Verweilen. 8. aktualisierte und erweiterte Ausgabe. Innsbruck.

Österreichischer Alpenverein (Hg.). 2018b. Kleine und feine Orte zum Genießen und Verweilen. Ein alpenkulinarisches Reisebuch (verfasst vom Roland Kals). 3. Aufl. Innsbruck.

Protokoll zur Durchführung der Alpenkonvention von $1991 \mathrm{im}$ Bereich Tourismus. Als pdf abrufbar unter: https://www.alpconv.org/fileadmin/user_upload/Convention/DE/Protocol_Tourism_DE.pdf.

Interview mit Peter Haßlacher am 13.05.2019, Innsbruck.

Interview mit dem Geschäftsführer der Tourismusinformation Ramsau am 07.05. 2019, Ramsau bei Berchtesgaden.

Interview mit dem Alpenvereinsobmann der Sektion Großarl-Hüttschlag und Gründer des Vereins BergGesund am 03.03. 2020, Hüttschlag. 
Siebenhaar, Hans-Peter. 2020. Reinhold Messner: „Wir müssen aus den Fehlentwicklungen in Ischgl lernen und umdenken". Handelsblatt vom 16.05.2020. Abrufbar unter: https://www.handelsblatt.com/arts_und_ style/lifestyle/bergsteiger-legende-reinhold-messner-wir-muessen-ausden-fehlentwicklungen-in-ischgl-lernen-und-umdenken/25832766. html?ticket=ST-1409598-R7cMPcwl5lIBg3MDa4cy-ap4 [Stand 23.05.2020].

Website der Bergsteigerdörfer. Abrufbar unter: https://www.bergsteigerdoerfer.org/ [Stand 05.06.2020].

Website der Bergsteigerdörfer: Die Philosophie der Bergsteigerdörfer. Abrufbar unter: https://www.bergsteigerdoerfer.org/6-0-Die-Philosphie-der-Bergsteigerdoerfer.html [Stand 22.05.2020].

\section{Literatur}

Antonietti, Thomas. 2000. Bauern. Bergführer. Hoteliers. Fremdenverkehr und Bauernkultur Zermatt und Aletsch 1850-1950. Baden: Hier und Jetzt.

Bahr, Hans-Dieter. 1997. Der Gast. In: Kammerhofer-Aggermann, Ulrike (Hg.): „Herzlich willkommen!“ Rituale der Gastlichkeit. (Salzburger Beiträge zur Volkskunde, 9). Salzburg: Salzburger Landesinstitut für Volkskunde: $35-46$.

Bausinger, Hermann. 1991. Grenzenlos... Ein Blick auf den modernen Tourismus. In: Bausinger, Hermann - Beyrer, Klaus - Korff, Gottfried (Hg.): Reisekultur. Von der Pilgerfahrt zum modernen Tourismus. München: Beck: 343-353.

Gyr, Ueli. 1992. Kultur für Touristen und Touristenkultur. Plädoyer für qualitative Analysen in der Reiseforschung. In: Kramer, Dieter - Lutz, Roland (Hg.): Reisen und Alltag. Beiträge zur kulturwissenschaftlichen Tourismusforschung. Frankfurt a.M.: Inst. für Kulturanthropologie und Europ. Ethnologie: 19-38.

Heimerdinger, Timo. 2005. Schmackhafte Symbole und alltägliche Notwendigkeit. Zu Stand und Perspektiven der volkskundlichen Nahrungsforschung. Zeitschrift für Volkskunde 101, 2: 205-218.

Hennig, Christoph. 1997. Reiselust. Touristen, Tourismus und Urlaubskultur. Frankfurt a.M. - Leipzig: Insel-Verl.

Lauterbach, Burkhart. 2006. Tourismus. Eine Einführung aus Sicht der volkskundlichen Kulturwissenschaft. Würzburg: Königshausen \& Neumann.

Leimgruber, Walter. 2005. Alpine Kultur: Welche Kultur für welchen Raum? In: Binder, Beate u.a. (Hg.): Ort. Arbeit. Körper. Ethnographie Europäischer Modernen (34. Kongress der Deutschen Gesellschaft für Volkskunde, Berlin 2003). New York - München - Berlin: Waxman: 147-155. 
Löfgren, Orvar. 1999. On Holiday. A History of Vacationing. Berkeley, Los Angeles - London: University of California Press.

Opaschowski, Horst W. 2001. Das gekaufte Paradies: Tourismus im 21. Jahrhundert. Hamburg: Germa Press.

Röthl, Martina. 2014. Tiroler Privatvermietung: „Und sie waren das alle so gewöhnt“. In: Rolshoven Johanna u.a. (Hg.): Mobilitäten! (Voyage. Jahrbuch für Reise- und Tourismusforschung, 10). Berlin: Metropol: 137-146.

Röthl, Martina. 2018. Über Institutionen und Personen „guten Willens“ Tourismus zwischen Gemeinwohl, Überzeugung und Einzelinteressen. In: Rolshoven, Johanna - Schneider, Ingo (Hg.): Dimensionen des Politischen. Ansprüche und Herausforderungen der Empirischen Kulturwissenschaft. Berlin: Neofelis Verlag: 399-407.

Scharfe, Martin. 2007. Berg-Sucht. Eine Kulturgeschichte des frühen Alpinismus 1750-1850. Wien u.a.: Böhlau.

Schrutka-Rechtenstamm, Adelheid. 1994. „Die Gäste fühlen sich wohl bei uns" - Begegnungen durch Tourismus. In: Pöttler, Burkhard (Hg.): Tourismus und Regionalkultur. (Buchreihe der Österreichischen Zeitschrift für Volkskunde, Bd.12). Wien: Verein für Volkskunde: 85-94.

Shore, Cris. 2012. (Why) Policy Matters. In: The SAGE Handbook of Social Anthropology. Anthropology and Public Policy. Auckland: 89-119.

Shore, Cris - Wright, Susan (Hg.). 1997. Anthropology of Policy: Perspectives on Governance and Power. London - New York: Routledge.

Stadelmann, Christian. 1994. „Was gond mi d'Gäscht a?!“ Zum Zusammenhang von Tourismus und regionaler Identität am Beispiel des Bregenzerwaldes. In: Pöttler, Burkhard (Hg.): Tourismus und Regionalkultur. (Buchreihe der Österreichischen Zeitschrift für Volkskunde, Bd.12). Wien: Verein für Volkskunde: 259-276.

Tschofen, Bernhard. 2010. Kulinaristik und Regionalkultur: Kulturwissenschaftliches zur "regionalen Küche" in Theorie und Praxis. Antares 3: $25-45$.

Ureta, Sebastian. 2014. Policy assemblages: proposing an alternative conceptual framework to study public action. Policy Studies 35, 3: 303-318.

Urry, John - Larsen, Jonas. 2011. The Tourist Gaze 3.0. London: Sage Publications: 1-30.

Wernhart, Karl R. 1997. Rituale der Gastlichkeit. Kulturanthropologische Universalien. In: Kammerhofer-Aggermann, Ulrike (Hg.): „Herzlich willkommen!" Rituale der Gastlichkeit. (Salzburger Beiträge zur Volkskunde, 9). Salzburg: Salzburger Landesinstitut für Volkskunde: 23-33. 